\title{
A Novel Approach for Ranking Intuitionisitc Fuzzy Numbers and its Application to Decision Making
}

\author{
Meishe Liang \\ Shijiazhuang Tiedao University \\ Ju-Sheng Mi ( $\nabla$ mijsh@263.net ) \\ Hebei Normal University https://orcid.org/0000-0002-3753-4490 \\ Shaopu Zhang \\ Shijiazhuang Tiedao University \\ Chenxia Jin \\ Hebei University of Science and Technology
}

\section{Research Article}

Keywords: Intuitionistic fuzzy number , Intuitionistic fuzzy set , Ideal measure, Multi-attribute decision making

Posted Date: July 6th, 2021

DOI: https://doi.org/10.21203/rs.3.rs-582033/v1

License: (1) (1) This work is licensed under a Creative Commons Attribution 4.0 International License. Read Full License 


\title{
A novel approach for ranking intuitionisitc fuzzy numbers and its application to decision making
}

\author{
Meishe Liang ${ }^{1,2} \cdot$ Jusheng $\mathrm{Mi}^{2 *} \cdot$ Shaopu Zhang ${ }^{1} \cdot$ Chenxia $\mathbf{J i n}^{2,3}$
}

Received: date / Accepted: date

\begin{abstract}
Ranking intuitionistic fuzzy numbers is an important issue in practical application of intuitionistic fuzzy sets. For making a rational decision, people need to get an effective sorting over the set of intuitionistic fuzzy numbers. Many scholars rank intuitionistic fuzzy numbers by defining different measures. These measures do not comprehensively consider the fuzzy semantics expressed by membership degree, nonmembership degree and hesitancy degree of intuitionistic fuzzy numbers. As a result, the ranking results are often counterintuitive, such as the indifference problems, the nonrobustness problems, etc. In this paper, according to geometrical representation, a novel measure for intuitionistic fuzzy number is defined, which is called the ideal measure. After that a new sorting approach of intuitionistic fuzzy numbers is proposed. It is proved that the intuitionistic fuzzy order obtained by the ideal measure satisfies the properties of weak admissibility,
\end{abstract}

\section{Meishe Liang}

E-mail: liangmeishe@163.com

Jusheng Mi

E-mail: mijsh@263.net

Shaopu Zhang

E-mail: shaopuzhang@hotmail.com

Chenxia Jin

E-mail: jinchenxia2005@126.com

${ }^{1}$ Department of Mathematics and Physics, Shijiazhuang Tiedao University,

Shijiazhuang 050043, P.R.China

${ }^{2}$ School of Mathematical Science, Hebei Normal University, Shijiazhuang 050024, P.R.China

${ }^{3}$ School of Economics and Management, Hebei University of Science and Technology,

Shijiazhuang 050018, P.R.China membership degree robustness, nonmembership degree robustness, and determinism. Numerical example is applied to illustrate the effectiveness and feasibility of this method. Finally, using the presented approach, the optimal alternative can be acquired in multi-attribute decision making problem. Comparison analysis shows that the intuitionistic fuzzy value ordering method obtained by the ideal measure is more effectiveness and simplicity than other existing methods.

Keywords Intuitionistic fuzzy number - Intuitionistic fuzzy set · Ideal measure · Multi-attribute decision making

\section{Introduction}

After the fuzzy set [31] was proposed by Zadeh in 1965, the mathematical research has been extended from the precise problems to the fuzzy problems. The essence of fuzzy set is to extend the membership value $\mu_{A}(x)$ of element $x$ for set $A$ from $\{0,1\}$ to any value on closed interval $[0,1]$. The value $1-\mu_{A}(x)$ is used to express the degree to which $x$ is not belong to the set $A$. As a direct extension of fuzzy set theory, intuitionistic fuzzy set (IFS) [2,3] was first introduced by Atanassov in 1986, in which membership degree, nonmembership degree and hesitancy degree are used to characterize the objective world in simultaneously. Therefore, it is more delicate in describing the fuzziness of the real wrold and more flexible in dealing with the uncertainty. At present, the theoretical and practical application of IF$\mathrm{S}$ have been deeply and attentively studied and have been widely used in many application fields [10,18,33], such as medicine diagnosis $[8,11]$, multi-attribute decision making $[9,20,34,38]$, cluster analysis $[5,19,36]$, 
feature extraction [21-23], pattern recognition [24,25] and other aspects.

With the in-depth study of IFS in the application field, many pioneering works have been focused on comparing and ranking intuitionistic fuzzy numbers (IFNs) [32]. A large number of scholars make great efforts in constructing different measures to rank IFSs (IFNs) and apply them to practical problems. $\mathrm{Xu}$ and Yager [30] firstly presented the rule to sort IFNs by two functions in 2006. They are score function and accuracy function. Comparing two IFNs, the larger the score function is, the higher order the IFN is. If the two IFNs have the same score function, the larger the accuracy function is, the higher order the IFN is. This method is simple and easy to operate. However, if there is a slight change of membership degree or nonmembership degree, the ranking results may be completely opposite. Taking into account both the reliability information and the amount of information represented by an IFN, Szmidt [26] constructed the measure $R$ to rank IFNs. However, some counterintuitive ranking results will be produced by measure $R$. Based on the drawback of the inadmissibility of IFNs order induced by measure $R$, Guo [13] proposed a new measure $Z_{Q}$ to rank IFNs by the view of the geometrical representation of IFNs. The method can also set different parameters to express different preference of decision makers. Based on the similarity measure of IFNs, Zhang [35] proposed the measure $l$ for sorting IFNs. In [27], Xing et al. pointed out that the order induced by measure $l$ could output unreasonable ordering results known as the determinacy problem. Base on the Euclidean distance and the geometrical representation of IFNs, Xing et al. [27] introduced the measure $P$ for sorting IFNs. This measurement takes into account the Euclidean distance between the ideal positive IFN and the IFNs of discussion. Xing et al. pointed out that the closer the IFN with the ideal positive IFN is, the more preference and the higher order of the IFN is. Although the rank of the IFNs induced by measure $P$ possesses a lot of attractive properties, this method does not consider the distance between the ideal negative IFN and the IFNs of discussion. Huang et al. [16] indicated that the farther the distance between the IFN and the ideal negative IFN is, the more favorable and the higher order of the corresponding IFN should be. In this view, the relativedistance-based favorable measure $M_{E}$ is defined to rank IFNs. For any two different IFNs, if the membership degree and the nonmembership degree have the same value respectively, we have the same measure $M_{E}$ value. However, the hesitation degree of the two IFNs are obviously different, and the hesitation degree indicates the degree of uncertainty, which is obviously unreasonable.

These methods mentioned above are important for ranking IFNs, and have been used in many practical problems. As we show in Section 3, the ranking results by the mentioned approaches are often counterintuitive, such as the indifference problems, the non-robustness problems, etc. It is necessary to present more effective and reasonable measure for sorting IFNs.

Based on the previous works, our purpose is to construct a new measure for sorting IFNs. This measure called ideal measure on the basis of Euclidean distance and the geometrical representation of IFNs. Some important properties are also addressed.

The remainder of the paper is structured as follows: In Section 2, we briefly review some preliminary notions and concepts, such as IFS, IFN, which will be used in the following content. In Section 3, we introduce some exiting methods to rank IFNs in detail. In Section 4, we introduce a novel approach for ranking IFNs. Meanwhile, some desirable properties have been also checked. After that, illustrate examples are applied to show the reasonability of the proposed method. Section 5 discusses the optimal alternative selecting in multi-attribute decision making problem. In Section 6, we conclude this paper by an outlook for further research.

\section{Preliminaries}

This section first briefly recalls some preliminary concepts, such as intuitionistic fuzzy set and intuitionistic fuzzy number.

Definition $1[2,3]$ An IF set $\mathbf{A}$ on the universe $X$ is defined by $\mathbf{A}=\left\{\left\langle\mu_{\mathbf{A}}(x), \nu_{\mathbf{A}}(x)\right\rangle: x \in X\right\}$, in which two mappings $\mu_{\mathbf{A}}: X \rightarrow[0,1]$ and $\nu_{\mathbf{A}}: X \rightarrow[0,1]$ represent the membership degree and nonmembership degree of the element $x \in X$ to $\mathbf{A}$. For any $x \in X$, $0 \leq \mu_{\mathbf{A}}(x)+\nu_{\mathbf{A}}(x) \leq 1$. We use $1-\mu_{\mathbf{A}}(x)-\nu_{\mathbf{A}}(x)$ to illustrate the hesitancy degree of $x \in X$ to $\mathbf{A}$ (namely $\left.\pi_{\mathbf{A}}(x)\right)$. Note that if for any $x \in X, \pi_{\mathbf{A}}(x)=0$, the IFS A degenerates into a fuzzy set. All IF subsets on $X$ is denoted by $\mathcal{I} \mathcal{F S}(X)$.

Definition $2[2,3]$ For two IFSs $\mathbf{A}=\left\{\left\langle\mu_{\mathbf{A}}(x), \nu_{\mathbf{A}}(x)\right\rangle\right.$ : $x \in X\}, \mathbf{B}=\left\{\left\langle\mu_{\mathbf{B}}(x), \nu_{\mathbf{B}}(x)\right\rangle: x \in X\right\}$. The operational law are defined as follows:

(1) $\mathbf{A} \subseteq \mathbf{B}$ if and only if $\mu_{A}(x) \leq \mu_{\mathbf{B}}(x), \nu_{\mathbf{A}}(x) \geq$ $\nu_{\mathbf{B}}(x), \forall x \in X$;

(2) $\mathbf{A}=\mathbf{B}$ if and only if $\mathbf{A} \subseteq \mathbf{B}, \mathbf{B} \subseteq \mathbf{A}$;

$(3) \sim \mathbf{A}=\left\{\left\langle\nu_{\mathbf{A}}(x), \mu_{\mathbf{A}}(x)\right\rangle: x \in X\right\}$;

(4) $\mathbf{A} \cap \mathbf{B}=\left\{\left\langle\mu_{\mathbf{A}}(x) \wedge \mu_{\mathbf{B}}(x), \nu_{\mathbf{A}}(x) \vee \nu_{\mathbf{B}}(x)\right\rangle: x \in\right.$ $X\}$; $X\}$.
(5) $\mathbf{A} \cup \mathbf{B}=\left\{\left\langle\mu_{\mathbf{A}}(x) \vee \mu_{\mathbf{B}}(x), \nu_{\mathbf{A}}(x) \wedge \nu_{\mathbf{B}}(x)\right\rangle: x \in\right.$ 
Definition 3 [28] Let $\mathbf{A}$ be an IFS on the universe $X$, for $x \in X, \mathbf{A}(x)=\left\langle\mu_{\mathbf{A}}(x), \nu_{\mathbf{A}}(x)\right\rangle$ is called an IFN (or IF value).

Definition 4 [29] Let $\mathbf{A}\left(x_{1}\right), \mathbf{A}\left(x_{2}\right)$ be two IFNs. Some operational law are defined as follows:

(1) $\mathbf{A}\left(x_{1}\right) \oplus \mathbf{A}\left(x_{2}\right)=\left\langle\mu_{\mathbf{A}}\left(x_{1}\right)+\mu_{\mathbf{A}}\left(x_{2}\right)-\mu_{\mathbf{A}}\left(x_{1}\right)\right.$. $\left.\mu_{\mathbf{A}}\left(x_{2}\right), \nu_{\mathbf{A}}\left(x_{1}\right) \cdot \nu_{\mathbf{A}}\left(x_{2}\right)\right\rangle$

(2) $\mathbf{A}\left(x_{1}\right) \otimes \mathbf{A}\left(x_{2}\right)=\left\langle\mu_{\mathbf{A}}\left(x_{1}\right) \cdot \mu_{\mathbf{A}}\left(x_{2}\right), \nu_{\mathbf{A}}\left(x_{1}\right)+\right.$ $\left.\nu_{\mathbf{A}}\left(x_{2}\right)-\nu_{\mathbf{A}}\left(x_{1}\right) \cdot \nu_{\mathbf{A}}\left(x_{2}\right)\right\rangle$;

(3) $\lambda \mathbf{A}\left(x_{1}\right)=\left\langle 1-\left(1-\mu_{\mathbf{A}}\left(x_{1}\right)\right)^{\lambda}, \nu_{\mathbf{A}}^{\lambda}\left(x_{1}\right)\right\rangle$;

(4) $\left(\mathbf{A}\left(x_{1}\right)\right)^{\lambda}=\left\langle\mu_{\mathbf{A}}^{\lambda}\left(x_{1}\right), 1-\left(1-\nu_{\mathbf{A}}\left(x_{1}\right)\right)^{\lambda}\right\rangle$.

These operations provide a useful way to aggregate IFNs.

According to Definition $2, \mathbf{A}^{*}(x)=\langle 1,0\rangle$ is the biggest IFN, then we denote $\mathbf{A}^{*}(x)$ as the ideal positive IFN [26], while $\sim \mathbf{A}^{*}(x)=\langle 0,1\rangle$ is called the ideal negative IFN. Let $\mathbf{A}\left(x_{1}\right), \mathbf{A}\left(x_{2}\right)$ be two IFNs, if $\mathbf{A}\left(x_{1}\right) \subseteq \mathbf{A}\left(x_{2}\right)$, then we note $\mathbf{A}\left(x_{1}\right) \preceq_{I F N} \mathbf{A}\left(x_{2}\right)$. The score function and the accuracy function are important measures and often applied to sort IFNs.

Definition $5[6,15]$ Let $\mathbf{A}(x)$ be an IFN, $S_{\mathbf{A}}(x)=$ $\mu_{\mathbf{A}}(x)-\nu_{\mathbf{A}}(x), H_{\mathbf{A}}(x)=\mu_{\mathbf{A}}(x)+\nu_{\mathbf{A}}(x)$ are commonly referred to the score function (namely $S_{\mathbf{A}}(x)$ ) and the accuracy function (namely $H_{\mathbf{A}}(x)$ ), respectively.

From Definition 5, It is clear that the bigger the value of $S_{\mathbf{A}}(x)$, the higher the relative support of the $\mathbf{A}(x)$; while the lager the value of $H_{\mathbf{A}}(x)$, the more information $\mathbf{A}(x)$ contains.

\section{The ranking principle of IFNs and Existing Methods}

In this section, we will introduce some exiting methods for ranking IFNs. In order to comprehend these methods, some terminologies related to sorting principle of IFNs need to be introduced first.

\subsection{The ranking principle of IFNs}

A preorder is a kind of binary relationship on some particular nonempty set in which the order satisfies reflective and transitive [17]. Let $\preceq$ be a preorder defined over set $P$, if $\preceq$ is comparable, then $(P, \preceq)$ is called a weak order or total preorder [1]. In addition, if a weak order $(P, \preceq)$ satisfies antisymmetry [17], then $(P, \preceq)$ is denoted as a total order or linear order. It is clear that elements may be fixed when these elements are sorted by a total preorder, but not in a total order. That is to say, a weak order (total preorder) is a extension of a total order (linear order). In the earlier work, Bustince and Fernandez [4] proposed the notion of admissible order to refine the orders of intervals. Simply said, if an order $\preceq$ on IFNs is a linear order and refines the order $\preceq_{I F N}$, then it is an admissible order. However, the orders obtained by some measures, including the measure $R$, the measure $l$ and so on are not admissible orders sometimes, since these measures are all single continuous functions. In order to redefine the order of IFNs, Xing et al. [27] revised the definition of the admissible order.

Definition 6 [27] Let $(I F N, \preceq)$ be a preordered set on the set of IFN. If the order $\preceq$ satisfies the following conditions:

(1) $\preceq$ is a weak order on IFN;

(2) $\mathbf{A}\left(x_{1}\right), \mathbf{A}\left(x_{2}\right) \in I F N$, if $\mathbf{A}\left(x_{1}\right) \preceq_{I F N} \mathbf{A}\left(x_{2}\right)$, then $\mathbf{A}\left(x_{1}\right) \preceq \mathbf{A}\left(x_{2}\right)$.

Then the order $\preceq$ is a weak admissible order.

In another word, if $\preceq$ is a weak admissible order, then $\preceq$ is not only a weak order, but also refines the order $\preceq_{I F N}$.

Intuitively, the ordering of IFN should have certain stability. It should allow a small change to the membership (and nonmembership) degree of the IFNs while the ordering remains unchanged. In other words, the order is both membership degree and nonmembership degree robust.

Definition 7 [27] Let $\prec$ be an order on IFN. A $\left(x_{1}\right)$, $\mathbf{A}\left(x_{2}\right) \in I F N$ are two IFNs, where $\mu_{\mathbf{A}}\left(x_{1}\right) \cdot \mu_{\mathbf{A}}\left(x_{2}\right) \neq 0$ and $\pi_{\mathbf{A}}\left(x_{1}\right) \cdot \pi_{\mathbf{A}}\left(x_{2}\right) \neq 0$. The order $\prec$ is called membership degree robust if $\mathbf{A}\left(x_{1}\right) \prec \mathbf{A}\left(x_{2}\right)$ implies that there exists $\varepsilon>0$ such that $\mathbf{A}^{\prime}\left(x_{1}\right) \prec \mathbf{A}\left(x_{2}\right)\left(\mathbf{A}\left(x_{1}\right) \prec\right.$ $\left.\mathbf{A}^{\prime}\left(x_{2}\right)\right)$, where $\mathbf{A}^{\prime}\left(x_{1}\right)=\left\langle\mu_{\mathbf{A}}\left(x_{1}\right)+\varepsilon, \nu_{\mathbf{A}}\left(x_{1}\right)\right\rangle\left(\mathbf{A}^{\prime}\left(x_{2}\right)=\right.$ $\left.\left\langle\mu_{\mathbf{A}}\left(x_{2}\right)-\varepsilon, \nu_{\mathbf{A}}\left(x_{2}\right)\right\rangle\right)$.

Definition 8 [27] Let $\prec$ be an order on IFN. $\mathbf{A}\left(x_{1}\right)$, $\mathbf{A}\left(x_{2}\right) \in I F N$ are two IFNs, where $\nu_{\mathbf{A}}\left(x_{1}\right) \cdot \nu_{\mathbf{A}}\left(x_{2}\right) \neq 0$ and $\pi_{\mathbf{A}}\left(x_{1}\right) \cdot \pi_{\mathbf{A}}\left(x_{2}\right) \neq 0$. The order $\prec$ is called nonmembership degree robust if $\mathbf{A}\left(x_{1}\right) \prec \mathbf{A}\left(x_{2}\right)$ implies that there exists $\varepsilon>0$ such that $\mathbf{A}^{\prime}\left(x_{1}\right) \prec \mathbf{A}\left(x_{2}\right)$ $\left(\mathbf{A}\left(x_{1}\right) \prec \mathbf{A}^{\prime}\left(x_{2}\right)\right)$, where $\mathbf{A}^{\prime}\left(x_{1}\right)=\left\langle\mu_{\mathbf{A}}\left(x_{1}\right), \nu_{\mathbf{A}}\left(x_{1}\right)-\right.$ $\varepsilon\rangle\left(\mathbf{A}^{\prime}\left(x_{2}\right)=\left\langle\mu_{\mathbf{A}}\left(x_{2}\right), \nu_{\mathbf{A}}\left(x_{2}\right)+\varepsilon\right\rangle\right)$.

Intuitively, people are used to be vagueness averse when facing fuzzy information and incomplete information $[32,37]$. Thus, if two IFNs with the same value of score function, the more accurate of the IFN, the more preferable it will be. Now, a definition is used to indicate this fact.

Definition 9 [27] Let $\prec$ be an order on IFN. A $\left(x_{1}\right)$, $\mathbf{A}\left(x_{2}\right) \in I F N$ are two IFNs. The order $\prec$ is determinate if $S_{\mathbf{A}}\left(x_{1}\right)=S_{\mathbf{A}}\left(x_{2}\right)$ and $H_{\mathbf{A}}\left(x_{1}\right)<H_{\mathbf{A}}\left(x_{2}\right)$ imply $\mathbf{A}\left(x_{1}\right) \prec \mathbf{A}\left(x_{2}\right)$.

\subsection{Existing methods for ranking IFNs}

Now, we first review some proposed methods for sorting IFNs, which are widely used in applications. 
- Xu and Yager's rule [30]:

Definition 10 Let $\mathbf{A}\left(x_{1}\right), \mathbf{A}\left(x_{2}\right) \in I F N$ be two IFNs.

(1) if $S\left(\mathbf{A}\left(x_{1}\right)\right)<S\left(\mathbf{A}\left(x_{2}\right)\right)$, then $\mathbf{A}\left(x_{1}\right)$ is smaller than $\mathbf{A}\left(x_{2}\right)$, denoted by $\mathbf{A}\left(x_{1}\right) \prec_{X Y} \mathbf{A}\left(x_{2}\right)$.

(2) if $S\left(\mathbf{A}\left(x_{1}\right)\right)=S\left(\mathbf{A}\left(x_{2}\right)\right)$ and $H\left(\mathbf{A}\left(x_{1}\right)\right)<H\left(\mathbf{A}\left(x_{2}\right)\right)$ then $\mathbf{A}\left(x_{1}\right)$ is smaller than $\mathbf{A}\left(x_{2}\right)$, denoted by $\mathbf{A}\left(x_{1}\right) \prec_{X Y}$ $\mathbf{A}\left(x_{2}\right)$.

(3) if $S\left(\mathbf{A}\left(x_{1}\right)\right)=S\left(\mathbf{A}\left(x_{2}\right)\right)$ and $H\left(\mathbf{A}\left(x_{1}\right)\right)=H\left(\mathbf{A}\left(x_{2}\right)\right)$ then $\mathbf{A}\left(x_{1}\right)$ and $\mathbf{A}\left(x_{2}\right)$ represent the same information, denoted by $\mathbf{A}\left(x_{1}\right) \sim_{X Y} \mathbf{A}\left(x_{2}\right)$.

According to Definition 6 , the order $\prec_{X Y}$ is weak admissible. It is also clear that $\prec_{X Y}$ is determinate by Definition 9. For two IFNs $\langle 0.4,0.2\rangle$ and $\langle 0.5,0.3\rangle$, we can easily verify that $\langle 0.4,0.2\rangle \prec_{X Y}\langle 0.5,0.3\rangle$. However, $\forall \varepsilon>0$, we have the following results:

(1) $\langle 0.5,0.3\rangle \prec_{X Y}\langle 0.4+\varepsilon, 0.2\rangle$ and $\langle 0.5-\varepsilon, 0.3\rangle \prec_{X Y}$ $\langle 0.4,0.2\rangle$;

(2) $\langle 0.5,0.3+\varepsilon\rangle \prec_{X Y}\langle 0.4,0.2\rangle$ and $\langle 0.5,0.3\rangle \prec_{X Y}$ $\langle 0.4,0.2-\varepsilon\rangle$;

These results reflect the fact that $\prec_{X Y}$ is neither membership degree robust nor nonmembership degree robust.

- Szmidt and Kacprzyk' rule [26]:

Definition 11 The measure $R$ of $\mathbf{A}(x)$ is defined as

$$
R(\mathbf{A}(x))=0.5\left(1+\pi_{\mathbf{A}}(x)\right) H^{3}\left(\mathbf{A}^{*}(x), \mathbf{A}(x)\right),
$$

where $\mathbf{A}^{*}(x)=\langle 1,0\rangle, H^{3}\left(\mathbf{A}^{*}(x), \mathbf{A}(x)\right)=0.5\left(\mid \mu_{\mathbf{A}^{*}}(x)-\right.$ $\left.\mu_{\mathbf{A}}(x)|+| \nu_{\mathbf{A}^{*}}(x)-\nu_{\mathbf{A}}(x)|+| \pi_{\mathbf{A}^{*}}(x)-\pi_{\mathbf{A}}(x) \mid\right)$.

Simplify the above formula, we have:

$R(\mathbf{A}(x))=0.5\left(1+\pi_{\mathbf{A}}(x)\right)\left(1-\mu_{\mathbf{A}}(x)\right)$.

Szmidt et al. pointed out that the $R(\mathbf{A}(x))$ reflects the "quality" of $\mathbf{A}(x)$. The lager value of $R(\mathbf{A}(x))$ is, the less preferable and the lower order $\mathbf{A}(x)$ is. In this view, the order $\preceq_{R}$ for ranking IFNs can be defined as follows:

Let $\mathbf{A}\left(x_{1}\right), \mathbf{A}\left(x_{2}\right) \in I F N$ be two IFNs. $\mathbf{A}\left(x_{1}\right) \preceq_{R}$ $\mathbf{A}\left(x_{2}\right)$ if and only if $R\left(\mathbf{A}\left(x_{1}\right)\right) \geq R\left(\mathbf{A}\left(x_{2}\right)\right)$.

It is easy to verify that $\preceq_{R}$ is both membership degree and nonmembership degree robust. According to Definition 11, for any two IFNs $\mathbf{A}\left(x_{1}\right)$ and $\mathbf{A}\left(x_{2}\right)$, if $S_{\mathbf{A}}\left(x_{1}\right)=S_{\mathbf{A}}\left(x_{2}\right)$ and $H_{\mathbf{A}}\left(x_{1}\right)<H_{\mathbf{A}}\left(x_{2}\right)$, then it is easy to verify that $R\left(\mathbf{A}\left(x_{1}\right)\right)>R\left(\mathbf{A}\left(x_{2}\right)\right)$ and $\mathbf{A}\left(x_{1}\right) \prec_{R}$ $\mathbf{A}\left(x_{2}\right)$. This means that the order $\prec_{R}$ is determinate. In addition, for two IFNs $\langle 0.4,0.6\rangle$ and $\langle 0.5,0.1\rangle$, it is clear that $\langle 0.4,0.6\rangle<_{I F N}\langle 0.5,0.1\rangle$. However, $R(\langle 0.5,0.1\rangle)=$ 0.35 and $R(\langle 0.4,0.6\rangle)=0.3$. It is clear that $R(\langle 0.5,0.1\rangle)\rangle$ $R(\langle 0.4,0.6\rangle)$ and $\langle 0.5,0.1\rangle \prec_{R}\langle 0.4,0.6\rangle$. This mean$\mathrm{s}$ that the order $\prec_{R}$ does not satisfy the property of weak admissibility.

- Guo' s rule [13]:
Definition 12 The measure $Z_{Q}$ of $\mathbf{A}(x)$ is defined as

$$
Z_{Q}(\mathbf{A}(x))=\left(1-\frac{k}{1+k} \pi_{\mathbf{A}}(x)\right)\left(\mu_{\mathbf{A}}(x)+\frac{1}{1+k} \pi_{\mathbf{A}}(x)\right),
$$

where $k$ is used to express the preference of the decision maker. Moreover, $k \in(0,1)$ indicates the optimistic attitude of the decision maker, while $k=1$ and $k>1$ describe the neutral and pessimistic attitudes of the decision maker, respectively. The order $\preceq Z_{Q}$ for ranking IFNs can be defined as follows:

Let $\mathbf{A}\left(x_{1}\right), \mathbf{A}\left(x_{2}\right) \in I F N$ be two IFNs. $\mathbf{A}\left(x_{1}\right) \preceq Z_{Q}$ $\mathbf{A}\left(x_{2}\right)$ if and only if $Z_{Q}\left(\mathbf{A}\left(x_{1}\right)\right) \leq Z_{Q}\left(\mathbf{A}\left(x_{2}\right)\right)$.

Similar to the order $\preceq_{R}$, the order $\preceq_{Z_{Q}}$ for ranking IFNs is both membership degree and nonmembership degree robust. In [27], Xing et al. pointed out that the order $\preceq_{Z_{Q}}$ may yield an unreasonable ordering result known as the indifference problem. Simply said, a more preferable IFN could be recognized as well as a less preferable one.

- Zhang and Xu's rule [35]:

Definition 13 The measure $l$ of $\mathbf{A}(x)$ is defined as

$$
l(\mathbf{A}(x))=1-\frac{H^{3}\left(\mathbf{A}^{*}(x), \mathbf{A}(x)\right)}{H^{3}\left(\mathbf{A}^{*}(x), \mathbf{A}(x)\right)+H^{3}\left(\sim \mathbf{A}^{*}(x), \mathbf{A}(x)\right)},
$$

where $\mathbf{A}^{*}(x)=\langle 1,0\rangle, H^{3}\left(\mathbf{A}^{*}(x), \mathbf{A}(x)\right)=0.5\left(\mid \mu_{\mathbf{A}^{*}}(x)-\right.$ $\left.\mu_{\mathbf{A}}(x)|+| \nu_{\mathbf{A}^{*}}(x)-\nu_{\mathbf{A}}(x)|+| \pi_{\mathbf{A}^{*}}(x)-\pi_{\mathbf{A}}(x) \mid\right)$.

Simplify the above formula, we have:

$l(\mathbf{A}(x))=\frac{1-\nu_{\mathbf{A}}(x)}{1+\pi_{\mathbf{A}}(x)}$.

The order $\preceq_{l}$ for ranking IFNs can be defined as follows:

Let $\mathbf{A}\left(x_{1}\right), \mathbf{A}\left(x_{2}\right) \in I F N$ be two IFNs. $\mathbf{A}\left(x_{1}\right) \preceq_{l}$ $\mathbf{A}\left(x_{2}\right)$ if and only if $l\left(\mathbf{A}\left(x_{1}\right)\right) \leq l\left(\mathbf{A}\left(x_{2}\right)\right)$.

According to the literature [27], the order $\preceq_{l}$ is weak admissible. We can also prove that the order $\preceq_{l}$ is membership robust and nonmembership robust. In addition, for two IFNs $\langle 0.4,0.6\rangle$ and $\langle 0.3,0.5\rangle$, It is clear that $\langle 0.3,0.5\rangle<_{I F N}\langle 0.4,0.6\rangle$. However, $l(\langle 0.3,0.5\rangle) \approx 0.42$ and $l(\langle 0.4,0.6\rangle)=0.4 . l(\langle 0.4,0.6\rangle)<l(\langle 0.3,0.5\rangle) \Rightarrow$ $\langle 0.4,0.6\rangle \prec_{l}\langle 0.3,0.5\rangle$. This means that the order $\prec_{l}$ does not satisfy the property of determinacy.

- Xing et al.'s rule [27]:

Definition 14 The measure $P$ of $\mathbf{A}(x)$ is defined as

$$
P(\mathbf{A}(x))=1-\sqrt{\frac{\left(1-\mu_{\mathbf{A}}(x)\right)^{2}+\nu_{\mathbf{A}}^{2}(x)}{2}} .
$$

With the aid of the measure $P$, the order $\preceq_{P}$ for ranking IFNs can be defined as follows:

Let $\mathbf{A}\left(x_{1}\right), \mathbf{A}\left(x_{2}\right) \in I F N$ be two IFNs. $\mathbf{A}\left(x_{1}\right) \preceq_{P}$ $\mathbf{A}\left(x_{2}\right)$ if and only if $P\left(\mathbf{A}\left(x_{1}\right)\right) \leq P\left(\mathbf{A}\left(x_{2}\right)\right)$.

In [27], it has been proved in detail that the order $\preceq_{P}$ satisfies weak admissible, determinacy, and both membership degree and nonmembership degree robust. 
- Huang et al.' s rule [16]:

Definition 15 The measure $M_{E}$ of $\mathbf{A}(x)$ is defined as

$$
M_{E}(\mathbf{A}(x))=\sqrt{\frac{\mu_{\mathbf{A}}^{2}(x)+\left(1-\nu_{\mathbf{A}}(x)\right)^{2}}{2}}-\sqrt{\frac{\left(1-\mu_{\mathbf{A}}(x)\right)^{2}+\nu_{\mathbf{A}}^{2}(x)}{2}} .
$$

The order $\preceq_{M_{E}}$ for sorting IFNs can be defined as follows:

Suppose that $\mathbf{A}\left(x_{1}\right), \mathbf{A}\left(x_{2}\right) \in I F N$ are two IFNs. $\mathbf{A}\left(x_{1}\right) \preceq_{M_{E}} \mathbf{A}\left(x_{2}\right)$ if and only if $M_{E}\left(\mathbf{A}\left(x_{1}\right)\right) \leq M_{E}\left(\mathbf{A}\left(x_{2}\right)\right)$.

Similar to the order $\preceq_{P}$, it can be proved that the order $\preceq_{M_{E}}$ satisfies weak admissible and both membership robust and nonmembership robust. However, the order $\preceq_{M_{E}}$ is not determinate. Let us consider the following example. For $\mathbf{A}(x) \in I F N$, if $\mu_{\mathbf{A}}(x)=\nu_{\mathbf{A}}(x) \leq$ 0.5 , then $M_{E}(\mathbf{A}(x)) \equiv 1$, such as $M_{E}(\langle 0.2,0.2\rangle)=$ $M_{E}(\langle 0.3,0.3\rangle)=M_{E}(\langle 0.5,0.5\rangle)=1$. Intuitively, this result is obviously unreasonable.

\section{A novel approach for ranking IFNs}

In order to make a reasonable ordering, we should have a deep understanding of the fuzzy semantics of membership degree, nonmembership degree and hesitation degree in IFNs. In fact, three factors should be considered simultaneously while ranking IFNs. Firstly, the closer the distance between one IFN with the ideal positive IFN is, the higher order of the IFN should be. Secondly, the farther the distance between one IFN with the ideal negative IFN is, the higher order of the IFN should be. Finally, the smaller the hesitancy degree is, the more information the IFN contains, and the higher order of the IFN should be. Now we provide a list of axioms that a "reasonable" measure of IFN should satisfy for ranking IFNs. These axioms are summarized as follows.

Definition 16 Denote $I: I F N \rightarrow[-1,1]$, then $I$ is called the ideal measure of IFN, if $\forall \alpha, \beta \in I F N$, it satisfies the following properties:

(1) $-1 \leq I(\alpha) \leq 1$;

(2) $I(\alpha)=1$ if and only if $\alpha=\langle 1,0\rangle$;

(3) $I(\alpha)=-1$ if and only if $\alpha=\langle 0,1\rangle$;

(4) $\beta \preceq_{I F N} \alpha \Rightarrow I(\beta) \leq I(\alpha)$.

According to Definition 16, we have a new measure as following.

Definition 17 Suppose $\alpha=\left\langle\mu_{\alpha}, \nu_{\alpha}\right\rangle$ is an IFN. Define the measure $I$ of IFN as:

$$
I(\alpha)=\sqrt{\frac{\mu_{\alpha}^{2}+\left(1-\nu_{\alpha}\right)^{2}}{2}}-\sqrt{\frac{\left(1-\mu_{\alpha}\right)^{2}+\nu_{\alpha}^{2}}{2}}-\frac{1-\mu_{\alpha}-\nu_{\alpha}}{2} .
$$

Theorem 1. $I$ is the ideal measure of IFN.
Proof (1) According to Definition 6, take the partial derivative of both sides of the measure $I$ with respect to $\mu_{\alpha}$,

$$
\begin{aligned}
I_{\mu_{\alpha}}^{\prime}(\alpha) & =\frac{1}{\sqrt{2}}\left(\frac{\mu_{\alpha}}{\sqrt{\mu_{\alpha}^{2}+\left(1-\nu_{\alpha}\right)^{2}}}+\frac{1-\mu_{\alpha}}{\sqrt{\left(1-\mu_{\alpha}\right)^{2}+\nu_{\alpha}^{2}}}+\frac{1}{\sqrt{2}}\right) \\
& >0 .
\end{aligned}
$$

It means that $I(\alpha)$ is monotone increasing with respect to $\mu_{\alpha}$.

Then take the partial derivative of both sides of the measure $I$ with respect to $\nu_{\alpha}$,

$$
\begin{aligned}
I_{\nu_{\alpha}}^{\prime}(\alpha) & =\frac{1}{\sqrt{2}}\left(-\frac{1-\nu_{\alpha}}{\sqrt{\mu_{\alpha}^{2}+\left(1-\nu_{\alpha}\right)^{2}}}-\frac{\nu_{\alpha}}{\sqrt{\left(1-\mu_{\alpha}\right)^{2}+\nu_{\alpha}^{2}}}+\frac{1}{\sqrt{2}}\right) \\
& =\frac{1}{\sqrt{2}}\left(\frac{1}{\sqrt{2}}-\frac{1-\nu_{\alpha}}{\sqrt{\mu_{\alpha}^{2}+\left(1-\nu_{\alpha}\right)^{2}}}-\frac{1-\nu_{\alpha}}{\sqrt{\left(1-\mu_{\alpha}\right)^{2}+\nu_{\alpha}^{2}}}\right) \\
& \leq \frac{1}{\sqrt{2}}\left(\frac{1}{\sqrt{2}}-\frac{\nu_{\alpha}}{\sqrt{\left(1-\nu_{\alpha}\right)^{2}+\left(1-\nu_{\alpha}\right)^{2}}}-\frac{\nu_{\alpha}}{\sqrt{\left(1-\mu_{\alpha}\right)^{2}+\nu_{\alpha}^{2}}}\right) \\
& =\frac{1}{\sqrt{2}}\left(\frac{1}{\sqrt{2}}-\frac{1-\nu_{\alpha}}{\sqrt{2}\left(1-\nu_{\alpha}\right)}-\frac{\nu_{\alpha}}{\sqrt{\left(1-\mu_{\alpha}\right)^{2}+\nu_{\alpha}^{2}}}\right) \\
& \leq \frac{1}{\sqrt{2}}\left(-\frac{\nu_{\alpha}}{\sqrt{\left(1-\mu_{\alpha}\right)^{2}+\nu_{\alpha}^{2}}}\right) \\
& \leq 0
\end{aligned}
$$

It is clear that $I(\alpha)$ is monotone decreasing with respect to $\nu_{\alpha}$.

Since $\forall \alpha \in I F N,\langle 0,1\rangle \preceq_{I F N}\left\langle 0, \nu_{\alpha}\right\rangle \preceq_{I F N}\left\langle\mu_{\alpha}, \nu_{\alpha}\right\rangle$ $\preceq_{I F N}\left\langle\mu_{\alpha}, 0\right\rangle \preceq_{I F N}\langle 1,0\rangle$. According to monotony, $I(\langle 0,1\rangle)$ $\leq I\left(\left\langle 0, \nu_{\alpha}\right\rangle\right) \leq I(\alpha) \leq I\left(\left\langle\mu_{\alpha}, 0\right\rangle\right) \leq I(\langle 1,0\rangle)$. According to Definition $17, I(\langle 0,1\rangle)=-1$ and $I(\langle 1,0\rangle)=1$. Thus, $\forall \alpha \in I F N,-1 \leq I(\alpha) \leq 1$.

It can be seen from (1) that both (2) and (3) are obvious.

(4) Suppose $\alpha=\left\langle\mu_{\alpha}, \nu_{\alpha}\right\rangle, \beta=\left\langle\mu_{\beta}, \nu_{\beta}\right\rangle$ are two IFNs. If $\beta \preceq_{I F N} \alpha$, we need to verify that $I(\beta) \leq I(\alpha)$. According to Definition $4, \mu_{\beta} \leq \mu_{\alpha}$ and $\nu_{\beta} \geq \nu_{\alpha}$. That is to say $\left\langle\mu_{\beta}, \nu_{\beta}\right\rangle \preceq_{I F N}\left\langle\mu_{\beta}, \nu_{\alpha}\right\rangle \preceq_{I F N}\left\langle\mu_{\alpha}, \nu_{\alpha}\right\rangle$. Since $I(\alpha)$ is monotone increasing w.r.t $\mu_{\alpha}$ and monotone decreasing w.r.t $\nu_{\alpha}$, respectively, we have $I\left(\left\langle\mu_{\beta}, \nu_{\beta}\right\rangle\right) \leq$ $I\left(\left\langle\mu_{\beta}, \nu_{\alpha}\right\rangle\right) \leq I\left(\left\langle\mu_{\alpha}, \nu_{\alpha}\right\rangle\right)$. Thus, $I(\beta) \leq I(\alpha)$.

In conclusion, $I$ is an ideal measure of IFN.

Lemma 1 Suppose $\alpha$ is an IFN. Then the ideal measure $I$ of the $\alpha$ is also represented as

$$
I(\alpha)=\sqrt{\frac{\mu_{\alpha}^{2}+\left(1-\nu_{\alpha}\right)^{2}}{2}}-\sqrt{\frac{\left(1-\mu_{\alpha}\right)^{2}+\nu_{\alpha}^{2}}{2}}-\frac{\pi_{\alpha}}{2} .
$$

Proof According to Definition 1, the proof is obvious.

Proposition 1 Suppose $\alpha$ is an IFN. If $\pi_{\alpha}=1-$ $\mu_{\alpha}-\nu_{\alpha}=0$, then we have $I(\alpha)=\mu_{\alpha}-\nu_{\alpha}$.

Proof If $\pi_{\alpha}=1-\mu_{\alpha}-\nu_{\alpha}=0$, then $\mu_{\alpha}=1-\nu_{\alpha}$ and $\nu_{\alpha}=1-\mu_{\alpha}$. Thus,

$$
\begin{aligned}
I(\alpha) & =\sqrt{\frac{\mu_{\alpha}^{2}+\left(1-\nu_{\alpha}\right)^{2}}{2}}-\sqrt{\frac{\left(1-\mu_{\alpha}\right)^{2}+\nu_{\alpha}^{2}}{2}}-\frac{\pi_{\alpha}}{2} \\
& =\sqrt{\frac{\mu_{\alpha}^{2}+\left(1-\nu_{\alpha}\right)^{2}}{2}}-\sqrt{\frac{\left(1-\mu_{\alpha}\right)^{2}+\nu_{\alpha}^{2}}{2}} \\
& =\sqrt{\frac{\mu_{\alpha}^{2}+\mu_{\alpha}^{2}}{2}}-\sqrt{\frac{\nu_{\alpha}^{2}+\nu_{\alpha}^{2}}{2}} \\
& =\mu_{\alpha}-\nu_{\alpha} .
\end{aligned}
$$


In fact, if $\pi_{\alpha}=1-\mu_{\alpha}-\nu_{\alpha}=0$, then the IFN $\alpha$ degenerates into a fuzzy number. $I(\alpha)=\mu_{\alpha}-\nu_{\alpha}=$ $2 \mu_{\alpha}-1$, the bigger membership degree of $\alpha$ is, the more favorable and the higher order $\alpha$ is, which is also a standard way for sorting fuzzy numbers.

According to the value of the ideal measure $I$, the method for sorting IFNs can be interpreted as follows:

Definition 18 Suppose $\alpha=\left\langle\mu_{\alpha}, \nu_{\alpha}\right\rangle, \beta=\left\langle\mu_{\beta}, \nu_{\beta}\right\rangle$ are two IFNs. $\beta \preceq_{I} \alpha$ if and only if $I(\beta) \leq I(\alpha)$.

According to Definition 16, the order induced by ${ }_{I}$ is weak order. In the following, we will verify whether the order for ranking IFNs induced by the ideal measure $I$ satisfies the properties of weak admissibility, membership degree robustness, nonmembership degree robustness, and determinacy.

Theorem 2. The order induced by $\preceq_{I}$ is weak admissibility.

Proof According to Definition 16 and Theorem 1, $\preceq_{I}$ is a weak admissible order on IFN.

Theorem 3. The order induced by $\prec_{I}$ is membership degree robust.

Proof Suppose that $\alpha=\left\langle\mu_{\alpha}, \nu_{\alpha}\right\rangle, \beta=\left\langle\mu_{\beta}, \nu_{\beta}\right\rangle$ are two IFNs, where $\mu_{\alpha} \cdot \mu_{\beta} \neq 0$ and $\pi_{\alpha} \cdot \pi_{\beta} \neq 0$. If $\alpha \prec_{I} \beta$, we only need to prove that there exists $\varepsilon>0$ such that $\alpha^{\prime} \prec_{I} \beta$ (and $\alpha \prec_{I} \beta^{\prime}$ ), where $\alpha^{\prime}=\left\langle\mu_{\alpha}+\varepsilon, \nu_{\alpha}\right\rangle, \beta^{\prime}=$ $\left\langle\mu_{\beta}-\varepsilon, \nu_{\beta}\right\rangle$. Let

$$
\begin{aligned}
f(\varepsilon) & =I(\beta)-I\left(\alpha^{\prime}\right) \\
& =\frac{1}{\sqrt{2}}\left(\sqrt{\mu_{\beta}^{2}+\left(1-\nu_{\beta}\right)^{2}}-\sqrt{\left(1-\mu_{\beta}\right)^{2}+\nu_{\beta}^{2}}-\frac{\pi_{\beta}}{\sqrt{2}}\right. \\
& \left.-\sqrt{\mu_{\alpha^{\prime}}^{2}+\left(1-\nu_{\alpha^{\prime}}\right)^{2}}+\sqrt{\left(1-\mu_{\alpha^{\prime}}\right)^{2}+\nu_{\alpha^{\prime}}^{2}}+\frac{\pi_{\alpha^{\prime}}}{\sqrt{2}}\right) \\
& =\frac{1}{\sqrt{2}}\left(\sqrt{\mu_{\beta}^{2}+\left(1-\nu_{\beta}\right)^{2}}-\sqrt{\left(1-\mu_{\beta}\right)^{2}+\nu_{\beta}^{2}}-\frac{\pi_{\beta}}{\sqrt{2}}\right. \\
& -\sqrt{\left(\mu_{\alpha}+\varepsilon\right)^{2}+\left(1-\nu_{\alpha}\right)^{2}}+\sqrt{\left(1-\mu_{\alpha}-\varepsilon\right)^{2}+\nu_{\alpha}^{2}} \\
& \left.+\frac{\pi_{\alpha}}{\sqrt{2}}-\frac{\varepsilon}{\sqrt{2}}\right)
\end{aligned}
$$

then

$$
\begin{aligned}
f^{\prime}(\varepsilon) & =\frac{-1}{\sqrt{2}}\left(\frac{1}{\sqrt{2}}+\frac{\mu_{\alpha}+\varepsilon}{\sqrt{\left(\mu_{\alpha}+\varepsilon\right)^{2}+\left(1-\nu_{\alpha}\right)^{2}}}+\frac{1-\mu_{\alpha}-\varepsilon}{\sqrt{\left(1-\mu_{\alpha}-\varepsilon\right)^{2}+\nu_{\alpha}^{2}}}\right) \\
& <0
\end{aligned}
$$

$f(\varepsilon)$ is strictly monotone decreasing w.r.t parameter $\varepsilon$. Since $f(0)=I(\beta)-I(\alpha)>0$, According to the function continuity properties, there exists $\varepsilon>0$ such that $f(\varepsilon)=\frac{f(0)}{2}>0$, which implies $\alpha^{\prime} \prec_{I} \beta$. Similarly, if $\alpha \prec_{I} \beta$ and $\beta^{\prime}=\left\langle\mu_{\beta}-\varepsilon, \nu_{\beta}\right\rangle$, then there exists $\varepsilon>0$ such that $\alpha \prec_{I} \beta^{\prime}$.

Theorem 4. The order $\prec_{I}$ is nonmembership degree robust.

Proof Suppose that $\alpha=\left\langle\mu_{\alpha}, \nu_{\alpha}\right\rangle, \beta=\left\langle\mu_{\beta}, \nu_{\beta}\right\rangle$ are two IFNs, where $\nu_{\alpha} \cdot \nu_{\beta} \neq 0$ and $\pi_{\alpha} \cdot \pi_{\beta} \neq 0$. If $\alpha \prec_{I} \beta$, we only need to prove that there exists $\varepsilon>0$ such that $\alpha^{\prime} \prec_{I} \beta$ (and $\alpha \prec_{I} \beta^{\prime}$ ), where $\alpha^{\prime}=\left\langle\mu_{\alpha}, \nu_{\alpha}-\varepsilon\right\rangle, \beta^{\prime}=$ $\left\langle\mu_{\beta}, \nu_{\beta}+\varepsilon\right\rangle$. Let

$$
\begin{aligned}
f(\varepsilon) & =I(\beta)-I\left(\alpha^{\prime}\right) \\
& =\frac{1}{\sqrt{2}}\left(\sqrt{\mu_{\beta}^{2}+\left(1-\nu_{\beta}\right)^{2}}-\sqrt{\left(1-\mu_{\beta}\right)^{2}+\nu_{\beta}^{2}}-\frac{\pi_{\beta}}{\sqrt{2}}\right. \\
& \left.-\sqrt{\mu_{\alpha^{\prime}}^{2}+\left(1-\nu_{\alpha^{\prime}}\right)^{2}}+\sqrt{\left(1-\mu_{\alpha^{\prime}}\right)^{2}+\nu_{\alpha^{\prime}}^{2}}+\frac{\pi_{\alpha^{\prime}}}{\sqrt{2}}\right) \\
& =\frac{1}{\sqrt{2}}\left(\sqrt{\mu_{\beta}^{2}+\left(1-\nu_{\beta}\right)^{2}}-\sqrt{\left(1-\mu_{\beta}\right)^{2}+\nu_{\beta}^{2}}-\frac{\pi_{\beta}}{\sqrt{2}}\right. \\
& -\sqrt{\mu_{\alpha}^{2}+\left(1-\nu_{\alpha}+\varepsilon\right)^{2}}+\sqrt{\left(1-\mu_{\alpha}\right)^{2}+\left(\nu_{\alpha}-\varepsilon\right)^{2}} \\
& \left.+\frac{\pi_{\alpha}}{\sqrt{2}}+\frac{\varepsilon}{\sqrt{2}}\right)
\end{aligned}
$$

Then

$$
\begin{aligned}
f^{\prime}(\varepsilon) & =\frac{1}{\sqrt{2}}\left(\frac{1}{\sqrt{2}}-\frac{1-\nu_{\alpha}+\varepsilon}{\sqrt{\mu_{\alpha}^{2}+\left(1-\nu_{\alpha}+\varepsilon\right)^{2}}}-\frac{\nu_{\alpha}-\varepsilon}{\sqrt{\left(1-\mu_{\alpha}\right)^{2}+\left(\nu_{\alpha}-\varepsilon\right)^{2}}}\right) \\
& <\frac{1}{\sqrt{2}}\left(\frac{1}{\sqrt{2}}-\frac{1-\nu_{\alpha}+\varepsilon}{\sqrt{2}\left(1-\nu_{\alpha}+\varepsilon\right)}-\frac{\nu_{\alpha}-\varepsilon}{\sqrt{\left(1-\mu_{\alpha}\right)^{2}+\left(\nu_{\alpha}-\varepsilon\right)^{2}}}\right) \\
& <0
\end{aligned}
$$

$f(\varepsilon)$ is strictly monotone decreasing w.r.t parameter $\varepsilon$. Since $f(0)=I(\beta)-I(\alpha)>0$, According to the function continuity properties, there exists $\varepsilon>0$ such that $f(\varepsilon)=\frac{f(0)}{2}>0$, which implies $\alpha^{\prime} \prec_{I} \beta$. Similarly, if $\alpha \prec_{I} \beta$ and $\beta^{\prime}=\left\langle\mu_{\beta}, \nu_{\beta}+\varepsilon\right\rangle$, then there exists $\varepsilon>0$ such that $\alpha \prec_{I} \beta^{\prime}$.

Theorem 5. The order induced by $\prec_{I}$ is determinate.

Proof Suppose that $\alpha=\left\langle\mu_{\alpha}, \nu_{\alpha}\right\rangle, \beta=\left\langle\mu_{\beta}, \nu_{\beta}\right\rangle$ are two IFNs, where $S(\beta)=S(\alpha)$ and $H(\beta)>H(\alpha)$. According to Definition 5 , we have $\mu_{\alpha}-\nu_{\alpha}=\mu_{\beta}-\nu_{\beta}$ and $\mu_{\beta}+\nu_{\beta}>$ $\mu_{\alpha}+\nu_{\alpha}$. In other words, $\mu_{\beta}-\mu_{\alpha}=\nu_{\beta}-\nu_{\alpha}, \mu_{\alpha}<\mu_{\beta}$, $\nu_{\alpha}<\nu_{\beta}$ and $\pi_{\beta}<\pi_{\alpha}$.

$$
\begin{aligned}
& \left(\mu_{\alpha}+\nu_{\alpha}+\mu_{\beta}+\nu_{\beta}-2\right)\left(\nu_{\beta}-\nu_{\alpha}\right)<0 \\
\Leftrightarrow & \left(\nu_{\beta}+\nu_{\alpha}\right)\left(\nu_{\beta}-\nu_{\alpha}\right)<\left(2-\mu_{\alpha}-\mu_{\beta}\right)\left(\nu_{\beta}-\nu_{\alpha}\right) \\
\Leftrightarrow & \left(\nu_{\beta}+\nu_{\alpha}\right)\left(\nu_{\beta}-\nu_{\alpha}\right)<\left(\mu_{\beta}-\mu_{\alpha}\right)\left(2-\mu_{\alpha}-\mu_{\beta}\right) \\
\Leftrightarrow & \left(\nu_{\beta}+\nu_{\alpha}\right)\left(\nu_{\beta}-\nu_{\alpha}\right)<\left(\left(1-\mu_{\alpha}\right)-\left(1-\mu_{\beta}\right)\right)\left(\left(1-\mu_{\alpha}\right)\right. \\
& \left.+\left(1-\mu_{\beta}\right)\right) \\
\Leftrightarrow & \nu_{\beta}^{2}-\nu_{\alpha}^{2}<\left(1-\mu_{\alpha}\right)^{2}-\left(1-\mu_{\beta}\right)^{2} \\
\Leftrightarrow & \left(1-\mu_{\beta}\right)^{2}+\nu_{\beta}^{2}<\left(1-\mu_{\alpha}\right)^{2}+\nu_{\alpha}^{2} \\
\Leftrightarrow & \sqrt{\frac{\left(1-\mu_{\beta}\right)^{2}+\nu_{\beta}^{2}}{2}}<\sqrt{\frac{\left(1-\mu_{\alpha}\right)^{2}+\nu_{\alpha}^{2}}{2}}
\end{aligned}
$$

On the other hand,

$$
\begin{aligned}
& \sqrt{\frac{\mu_{\beta}^{2}+\left(1-\nu_{\beta}\right)^{2}}{2}}-\frac{\pi_{\beta}}{2}-\sqrt{\frac{\mu_{\alpha}^{2}+\left(1-\nu_{\alpha}\right)^{2}}{2}}+\frac{\pi_{\alpha}}{2} \\
& =\sqrt{\frac{\mu_{\beta}^{2}+\left(1-\nu_{\beta}\right)^{2}}{2}}-\frac{1-\mu_{\beta}-\nu_{\beta}}{2}-\sqrt{\frac{\mu_{\alpha}^{2}+\left(1-\nu_{\alpha}\right)^{2}}{2}}+\frac{1-\mu_{\alpha}-\nu_{\alpha}}{2} \\
& =\frac{1}{\sqrt{2}}\left(\sqrt{\mu_{\beta}^{2}+\left(1-\nu_{\beta}\right)^{2}}-\sqrt{\mu_{\alpha}^{2}+\left(1-\nu_{\alpha}\right)^{2}}+\sqrt{2}\left(\mu_{\beta}-\mu_{\alpha}\right)\right) \\
& =\frac{1}{\sqrt{2}}\left(\sqrt{2}\left(\mu_{\beta}-\mu_{\alpha}\right)-\frac{\left(\mu_{\beta}-\mu_{\alpha}\right)\left(2-\mu_{\alpha}-\nu_{\alpha}-\mu_{\beta}-\nu_{\beta}\right)}{\sqrt{\mu_{\beta}^{2}+\left(1-\nu_{\beta}\right)^{2}}+\sqrt{\mu_{\alpha}^{2}+\left(1-\nu_{\alpha}\right)^{2}}}\right) \\
& =\frac{\mu_{\beta}-\mu_{\alpha}}{\sqrt{2}}\left(\sqrt{2}-\frac{\pi_{\alpha}+\pi_{\beta}}{\sqrt{\mu_{\beta}^{2}+\left(1-\nu_{\beta}\right)^{2}}+\sqrt{\mu_{\alpha}^{2}+\left(1-\nu_{\alpha}\right)^{2}}}\right) \\
& =\frac{\mu_{\beta}-\mu_{\alpha}}{\sqrt{2}}\left(\sqrt{2}-\frac{\pi_{\alpha}+\pi_{\beta}}{\sqrt{\mu_{\beta}^{2}+\left(\mu_{\beta}^{2}+\pi_{\beta}\right)^{2}}+\sqrt{\mu_{\alpha}^{2}+\left(\mu_{\alpha}^{2}+\pi_{\alpha}\right)^{2}}}\right) \\
& \geq \frac{\mu_{\beta}-\mu_{\alpha}}{\sqrt{2}}\left(\sqrt{2}-\frac{\pi_{\alpha}+\pi_{\beta}}{\pi_{\alpha}+\pi_{\beta}}\right) \\
& \geq 0
\end{aligned}
$$


Thus,

$\quad \frac{\sqrt{\frac{\mu_{\beta}^{2}+\left(1-\nu_{\beta}\right)^{2}}{2}}}{2}-\sqrt{\frac{\left(1-\mu_{\beta}\right)^{2}+\nu_{\beta}^{2}}{2}}-\frac{\pi_{\beta}}{2}>\sqrt{\frac{\mu_{\alpha}^{2}+\left(1-\nu_{\alpha}\right)^{2}}{2}}-$
$\sqrt{\frac{\left(1-\mu_{\alpha}\right)^{2}+\nu_{\alpha}^{2}}{2}}-\frac{\pi_{\alpha}}{2}$

From aforementioned process, we have $\alpha \prec_{I} \beta$, which concludes the proof.

Now, a numerical example is applied to to demonstrate the reasonability of the ideal measure.

Example 1. Suppose that there are eight different treatments $\left\{\mathbf{A}_{1}, \mathbf{A}_{2}, \cdots, \mathbf{A}_{8}\right\}$ for one patient. The treatments are evaluated by IFNs.Let $\mathbf{A}_{1}=\langle 0.30,0.30\rangle$, $\mathbf{A}_{2}=\langle 0.50,0.50\rangle, \mathbf{A}_{3}=\langle 0.39,0.50\rangle, \mathbf{A}_{4}=\langle 0.39,0.60\rangle$, $\mathbf{A}_{5}=\langle 0.673,0.0\rangle, \mathbf{A}_{6}=\langle 0.70,0.30\rangle, \mathbf{A}_{7}=\langle 0.40,0.0\rangle$, $\mathbf{A}_{8}=\left\langle 1-\frac{\sqrt{2}}{2}, 0.0\right\rangle$, respectively. Specifically speaking, treatment $\mathbf{A}_{1}=\langle 0.30,0.30\rangle$ influences $30 \%$ in a positive way for the symptoms, $30 \%$ in a negative way for the symptoms, and $40 \%$ in a case of unknown. So do the other treatments. Different measures are used to process these IFNs, the results are summarized in Table 1.

According to measure $R$, we have $R\left(\mathbf{A}_{6}\right)<R\left(\mathbf{A}_{5}\right)<$ $R\left(\mathbf{A}_{2}\right)<R\left(\mathbf{A}_{4}\right)<R\left(\mathbf{A}_{3}\right)<R\left(\mathbf{A}_{7}\right)<R\left(\mathbf{A}_{1}\right)<R\left(\mathbf{A}_{8}\right)$. Then the order of these IFNs is $\mathbf{A}_{8} \prec_{R} \mathbf{A}_{1} \prec_{R} \mathbf{A}_{7} \prec_{R}$ $\mathbf{A}_{3} \prec_{R} \mathbf{A}_{4} \prec_{R} \mathbf{A}_{2} \prec_{R} \quad \mathbf{A}_{5} \prec_{R} \mathbf{A}_{6}$. However, $\mathbf{A}_{1} \prec_{R}$ $\mathbf{A}_{3}$, which means the order induced by $\preceq_{R}$ is not a weak admissible order. According to measure $Z$, let $k=1$, we have $Z\left(\mathbf{A}_{3}\right)<Z\left(\mathbf{A}_{4}\right)<Z\left(\mathbf{A}_{1}\right)<Z\left(\mathbf{A}_{8}\right)<Z\left(\mathbf{A}_{7}\right)<$ $Z\left(\mathbf{A}_{2}\right)<Z\left(\mathbf{A}_{5}\right)=Z\left(\mathbf{A}_{6}\right)$. Then the order of these IFNs is $\mathbf{A}_{3} \prec_{Z} \mathbf{A}_{4} \prec_{Z} \quad \mathbf{A}_{1} \prec_{Z} \quad \mathbf{A}_{8} \prec_{Z} \quad \mathbf{A}_{7} \prec_{Z} \quad \mathbf{A}_{2} \prec_{Z}$ $A_{5} \approx_{Z} A_{6}$. However, $A_{7} \prec_{Z} A_{2}$, which means that the order induced by $\preceq_{Z}$ is not determinate. According to measure $l$, we have $l\left(\mathbf{A}_{4}\right)<l\left(\mathbf{A}_{3}\right)<l\left(\mathbf{A}_{1}\right)=$ $l\left(\mathbf{A}_{2}\right)<l\left(\mathbf{A}_{8}\right)<l\left(\mathbf{A}_{7}\right)<l\left(\mathbf{A}_{5}\right)=l\left(\mathbf{A}_{6}\right)$. Then the order of these IFNs is $\mathbf{A}_{4} \prec_{l} \quad \mathbf{A}_{3} \prec_{l} \quad \mathbf{A}_{1} \approx_{l} \quad \mathbf{A}_{2} \prec_{l}$

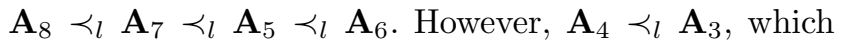
means that the order induced by $\preceq_{l}$ is not determinate. According to measure $P$, we have $P\left(\mathbf{A}_{3}\right)<P\left(\mathbf{A}_{4}\right)<$ $P\left(\mathbf{A}_{1}\right)<P\left(\mathbf{A}_{2}\right)=P\left(\mathbf{A}_{8}\right)<P\left(\mathbf{A}_{7}\right)<P\left(\mathbf{A}_{6}\right)=$ $P\left(\mathbf{A}_{5}\right)$. Then the order of these IFNs is $\mathbf{A}_{3} \prec_{P} \mathbf{A}_{4} \prec_{P}$

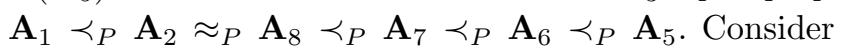
the distance between $\mathbf{A}_{2}$ and the ideal negative IFN $\langle 0,1\rangle$, the distance between $\mathbf{A}_{8}$ and the ideal negative IFN $\langle 0,1\rangle$, respectively. Then, we believe that $\mathbf{A}_{8}$ is more favorable than $\mathbf{A}_{2}$. According to measure $M_{E}$, we have $M_{E}\left(\mathbf{A}_{3}\right)<M_{E}\left(\mathbf{A}_{4}\right)<M_{E}\left(\mathbf{A}_{1}\right)=M_{E}\left(\mathbf{A}_{2}\right)<$ $M_{E}\left(\mathbf{A}_{8}\right)<M_{E}\left(\mathbf{A}_{7}\right)<M_{E}\left(\mathbf{A}_{6}\right)=M_{E}\left(\mathbf{A}_{5}\right)$. Then the order of these IFNs is $\mathbf{A}_{3} \prec_{M_{E}} \quad \mathbf{A}_{4} \prec_{M_{E}} \quad \mathbf{A}_{1} \approx_{M_{E}}$ $\mathbf{A}_{2} \prec_{M_{E}} \quad \mathbf{A}_{8} \prec_{M_{E}} \quad \mathbf{A}_{7} \prec_{M_{E}} \quad \mathbf{A}_{6} \prec_{M_{E}} \quad \mathbf{A}_{5}$. However, $\mathbf{A}_{1} \approx_{M_{E}} \mathbf{A}_{2}$, which means that the order $\preceq_{M_{E}}$ is not determinate. Comparing the ranking results of the IFNs induced by those measures, the ranking induced by ideal measure $I$ is more reasonable.
Table 1 Treatment evaluation using different measures

\begin{tabular}{ccccccc}
\hline$I F N$ & $R$ & $Z$ & $l$ & $P$ & $M$ & $I$ \\
\hline $\mathbf{A}_{1}$ & 0.490 & 0.400 & 0.500 & 0.461 & 0.000 & -0.200 \\
$\mathbf{A}_{2}$ & 0.250 & 0.500 & 0.500 & 0.500 & 0.000 & 0.000 \\
$\mathbf{A}_{3}$ & 0.430 & 0.354 & 0.413 & 0.386 & -0.205 & -0.310 \\
$\mathbf{A}_{4}$ & 0.308 & 0.393 & 0.396 & 0.395 & -0.210 & -0.215 \\
$\mathbf{A}_{5}$ & 0.217 & 0.700 & 0.754 & 0.769 & 0.621 & 0.458 \\
$\mathbf{A}_{6}$ & 0.150 & 0.700 & 0.700 & 0.700 & 0.400 & 0.400 \\
$\mathbf{A}_{7}$ & 0.480 & 0.490 & 0.625 & 0.576 & 0.337 & 0.037 \\
$\mathbf{A}_{8}$ & 0.604 & 0.418 & 0.586 & 0.500 & 0.237 & -0.117 \\
\hline
\end{tabular}

\section{IF multi-attribute decision making based on ideal measure}

\subsection{Problem description}

Multi-attribute decision making (MADM) is widespread in all areas of human social life, such as politics, economies, medical science, etc.. According to the information contained by different attributes, decision makers (DMs) need to sort the alternatives of the discourse or select the optimal alternative form the universe of discourse. Here, we consider an evaluation example of house selling taken from [20], which is described as follows.

There are six alternative houses for sale, denoted by $U=\left\{H_{1}, H_{2}, \cdots, H_{6}\right\}$. Let $A T=\left\{a_{1}, a_{2}, a_{3}, a_{4}\right\}$ be a set of decision parameters, including location, property service, building structure and price. For each alternative house $H_{i}(i \leq 6)$ and each attribute $a_{l}(l \leq 4)$, there is an intuitionistic fuzzy numbers denoted as $\mathbf{A}_{\mathbf{l}}\left(H_{i}\right)=$ $\left\langle\mu_{l}\left(H_{i}\right), \nu_{l}\left(H_{i}\right)\right\rangle$. The IFNs are used to describe the decision parameters of the houses, which is more close and accurate to the reality problem.

Table 2 The IFNs of the houses according to $A T$

\begin{tabular}{|c|c|c|c|c|}
\hline houses & $a_{1}$ & $u_{2}$ & $a_{3}$ & $a_{4}$ \\
\hline$H_{1}$ & $\langle 0.80,0.20\rangle$ & $\langle 0.78,0.18\rangle$ & $\langle 0.69,0.28\rangle$ & $\langle 0.78,0.12\rangle$ \\
\hline $\mathrm{H}_{2}$ & $\langle 0.76,0.23\rangle$ & $\langle 0.78,0.20\rangle$ & $\langle 0.70,0.28\rangle$ & $\langle 0.75,0.18\rangle$ \\
\hline$H_{3}$ & $\langle 0.80,0.10\rangle$ & $\langle 0.60,0.24\rangle$ & $\langle 0.75,0.20\rangle$ & $\langle 0.70,0.25\rangle$ \\
\hline$H_{4}$ & $\langle 0.75,0.20\rangle$ & $\langle 0.82,0.15\rangle$ & $\langle 0.55,0.28\rangle$ & $\langle 0.80,0.18\rangle$ \\
\hline$H_{5}$ & $\langle 0.65,0.28\rangle$ & $\langle 0.74,0.20\rangle$ & $\langle 0.70,0.15\rangle$ & $\langle 0.75,0.20\rangle$ \\
\hline$H_{6}$ & $\langle 0.80,0.10\rangle$ & $\langle 0.60,0.24\rangle$ & $\langle 0.75,0.15\rangle$ & $\langle 0.70,0.25\rangle$ \\
\hline
\end{tabular}

To maker our paper easier to understand, the IFNs of the decision parameters are shown in Table 2. The six houses need to be ranked completely according to the quality level of the four attributes.

\subsection{Decision-making information integration}

To solve the ranking problem above, there are two kinds of sorting process for decision makers to choose. The detail process is described as follows.

\section{Ranking method 1}

Step 1 From Definition 4, aggregate the decisionmaking information of different alternative houses. We 
Table 3 The aggregation information of each house

\begin{tabular}{ccccccc}
\hline & $H_{1}$ & $H_{2}$ & $H_{3}$ & $H_{4}$ & $H_{5}$ & $H_{6}$ \\
\hline $\mathbf{A A}\left(H_{i}\right)$ & $\langle 0.766,0.187\rangle$ & $\langle 0.749,0.219\rangle$ & $\langle 0.722,0.186\rangle$ & $\langle 0.748,0.197\rangle$ & $\langle 0.713,0.203\rangle$ & $\langle 0.722,0.173\rangle$ \\
\hline
\end{tabular}

denote the aggregating of the decision-making information as $\mathbf{A A}\left(H_{i}\right)=\oplus_{l=1}^{4} \frac{1}{4} \mathbf{A}_{\mathbf{l}}\left(H_{i}\right)$.

Step 2 According to Definition 16, calculate the ideal measure of the aggregation information $I\left(\mathbf{A A}\left(H_{i}\right)\right)$ for each alternative house.

Step 3 Compare the ideal measure and rank the alternative houses.

Example 2 According to ranking method one, we aggregate the decision-making information of alternative houses as shown in Table 3.

In terms of the computing the ideal measure of the aggregation information, the results is shown in Table 4.

Table 4 The ideal measure of the aggregation information

\begin{tabular}{ccccccc}
\hline & $H_{1}$ & $H_{2}$ & $H_{3}$ & $H_{4}$ & $H_{5}$ & $H_{6}$ \\
\hline$I\left(\mathbf{A A}\left(H_{i}\right)\right)$ & 0.555 & 0.514 & 0.486 & 0.522 & 0.465 & 0.492 \\
\hline
\end{tabular}

Then we rank the alternative houses according to the ideal measure. The ranking result we get from Step 3 is $H_{1}>H_{4}>H_{2}>H_{6}>H_{3}>H_{5}$.

\section{Ranking method 2}

Step 1 According to Definition 16, calculate the ideal measure of IFNs under different attributes $I\left(\mathbf{A}_{\mathbf{l}}\left(H_{i}\right)\right)$ for each alternative house.

Step 2 Aggregate the ideal measure of IFNs under different attributes for different alternative houses. We denote the aggregating ideal measure as $A I\left(H_{i}\right)=$ $\frac{1}{4} \Sigma_{l=l}^{4} I\left(\mathbf{A}_{\mathbf{l}}\left(H_{i}\right)\right)$.

Step 3 Compare the aggregation information and rank the alternative houses.

Example 3 According to Definition 16, the ideal measure of IFNs under different attributes for each alternative house are calculated as follows.

Table 5 The ideal measure of IFNs

\begin{tabular}{lllll}
\hline & $I\left(a_{1}\right)$ & $I\left(a_{2}\right)$ & $I\left(a_{3}\right)$ & $I\left(a_{4}\right)$ \\
\hline$H_{1}$ & 0.600 & 0.579 & 0.395 & 0.604 \\
$H_{2}$ & 0.525 & 0.570 & 0.410 & 0.532 \\
$H_{3}$ & 0.643 & 0.275 & 0.524 & 0.424 \\
$H_{4}$ & 0.524 & 0.654 & 0.181 & 0.610 \\
$H_{5}$ & 0.334 & 0.509 & 0.466 & 0.524 \\
$H_{6}$ & 0.643 & 0.275 & 0.545 & 0.424 \\
\hline
\end{tabular}

In terms of the aggregating the ideal measure of IFNs under different attributes, the results is shown in Table 6 .

Then we rank the alternative houses according to the ideal measure. The ranking result we get from Step 3 is $H_{1}>H_{2}>H_{4}>H_{6}>H_{3}>H_{5}$.
Table 6 The aggregation of the ideal measure

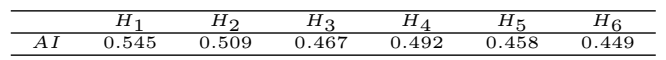

Note that the the sorting result we obtained above does not consider the decision makers' preference towards different attributes. Suppose the main consideration of the DMs is good education resources. The DMs insist that the location of the house is more important than other factors. Or, if the decision maker puts the dwelling quality in the first place, the property service will be more important than other factors. In this case, decision makers can set different weights to different attributes according to the preference. Suppose that the weights to different attributes are $\omega=\left\{\omega_{1}, \omega_{2}, \omega_{3}, \omega_{4}\right\}$, in Ranking method 1, we set the aggregating of the decision-making information $\mathbf{A} \mathbf{A}\left(H_{i}\right)=\oplus_{l=1}^{4} \omega_{l} \mathbf{A} \mathbf{l}\left(H_{i}\right)$. in Ranking method 2, we set the aggregating of the ideal measure of IFNs under different attributes $A I\left(H_{i}\right)=$ $\Sigma_{l=l}^{4} \omega_{l} I\left(\mathbf{A}_{\mathbf{l}}\left(H_{i}\right)\right)$.

Example 4 At this moment, two sets of weights are fixed to different attribute. They are $\omega^{1}=\{0.4,0.2,0.2,0.2\}$ and $\omega^{2}=\{0.5,0.1,0.2,0.2\}$. Similarly, we can obtain the ranking of the alternative houses, which are all summarized as following.

By Ranking method 1:

$$
\begin{aligned}
& \omega^{1}: H_{1}>H_{6}>H_{3}>H_{4}>H_{2}>H_{5}, \\
& \omega^{2}: H_{1}>H_{6}>H_{3}>H_{2}>H_{4}>H_{5} . \\
& \text { By Ranking method 2: } \\
& \omega^{1}: H_{1}>H_{2}>H_{6}>H_{3}>H_{4}>H_{5}, \\
& \omega^{2}: H_{1}>H_{6}>H_{3}>H_{2}>H_{4}>H_{5} .
\end{aligned}
$$

\subsection{Comparisons with related works}

In this subsection, comparison analysis is employed to illustrate the effectiveness of the proposed method.

Example 5 According to literature [37], the main MCDM ranking process can be explained as Max\{choicevalue $\}$ - Min\{hesitation $\}$ - Max\{score $\}$, in which different pairs of level values are used to sort the alternative houses. In detail, they are $L(0.70,0.30)$-level, $L$ (mid, mid)-level, L(top, bot)-level, L(top, top $)$-level, and $L(b o t, b o t)-$ level. Now, we compute and compare the level soft set $L(s, t)$ of each alternative house, the results are all summarized as we can see in Table 7 .

Then, rank the alternative houses by the choicevalue. The ranking result of the alternative houses is as following. 
Table 7 The choice-value of different level soft set $L(\mathrm{~s}, \mathrm{t})$

\begin{tabular}{cccccc}
\hline$U$ & $L(0.70,0.30)$ & $L(\mathrm{mid}, \mathrm{mid})$ & $L($ top, bot $)$ & $L$ (top,top $)$ & $L($ bot, bot $)$ \\
\hline$H_{1}$ & 4 & 2 & 0 & 1 & 1 \\
$H_{2}$ & 3 & 2 & 0 & 0 & 1 \\
$H_{3}$ & 3 & 2 & 1 & 2 & 1 \\
$H_{4}$ & 3 & 2 & 1 & 2 & 1 \\
$H_{5}$ & 3 & 3 & 0 & 0 & 2 \\
$H_{6}$ & 2 & 2 & 2 & 2 & 2 \\
\hline
\end{tabular}

$L(0.70,0.30): H_{2}>H_{1}=H_{3}=H_{4}=H_{5}>H_{6} ;$

$L($ mid, mid $): H_{5}>H_{1}=H_{2}=H_{3}=H_{4}=H_{6}$;

$L($ top, bot $): H_{6}>H_{3}=H_{4}>H_{1}=H_{2}=H_{5}$;

$L($ top, top $): H_{3}=H_{4}=H_{6}>H_{1}>H_{2}=H_{5}$;

$L($ bot, bot $): H_{6}>H_{1}=H_{3}=H_{4}=H_{5}>H_{2}$.

We cannot rank the alternative houses in a completely order only by the principle of the maximal choicevalue. Hence, it is necessary to calculate and compare the hesitation degree of the IFNs for all alternative houses. The result is shown in Table 8 .

Table 8 The hesitation degree of the alternative houses

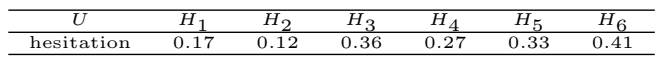

Next, we use the principle of Min\{hesitation\} on the basis of the maximal choice-value principle, the ranking result of the alternative houses is as follows.

$L(0.70,0.30): H_{2}>H_{1}>H_{4}>H_{5}>H_{3}>H_{6}$;

$L($ mid, $m i d): H_{5}>H_{2}>H_{1}>H_{4}>H_{3}>H_{6}$;

$L($ top, bot $): H_{6}>H_{4}>H_{3}>H_{2}>H_{1}>H_{5}$;

$L$ (top, top $): H_{4}>H_{3}>H_{6}>H_{1}>H_{2}>H_{5}$;

$L($ bot, bot $): H_{6}>H_{1}>H_{4}>H_{5}>H_{3}>H_{2}$.

At this moment, we sort the alternative houses in a strict order in all pairs of level values, and also get the optimal object.

According to Table 2, since $\mathbf{A}_{\mathbf{l}}\left(H_{3}\right) \subseteq \mathbf{A}_{\mathbf{l}}\left(H_{6}\right)$ for each attribute, we should get $H_{6}>H_{3}$ in all ranking results. However, we see that $H_{3}>H_{6}$ in the results of the level values $L(0.70,0.30), L(t o p, t o p)$ and $L($ mid, mid $)$. By contrast, this case does not appear in our method. In the other hand, the IFNs of $H_{1}$ is bigger than $H_{2}$ with respect to attributes $a_{1}, a_{2}$ and $a_{4}$. The IFN of $a_{3}$ for $H_{1}$ is nearly as the same as $H_{2}$. Intuitively, $H_{1}$ should be in front of $H_{2}$ all the ranked results. However, we see that $H_{2}>H_{1}$ in the results of the level values of $L(0.70,0.30), L($ top, bot $)$ and $L($ mid, mid $)$. As a matter of fact, our approach gets the order $H_{1}>H_{2}$, as desired.

Example 6 According to literature [20], multi-adjoint theory and Dempster-Shafer evidence theory are applied to tackle the multi-attribute group decision making problem. The main MADM ranking process can be explained as Belief structure construction-Evidence combination-Belief internal comparison. On the basis of different adjoint triples, the decision makers can obtain different results.

For all $i, j \leq 4, \tau: a_{i} \times a_{j} \rightarrow$ product:

$H_{6}>H_{3}>H_{1}>H_{4}>H_{5}>H_{2}$;

If $i, j \in\{2,3\}, \tau: a_{i} \times a_{j} \rightarrow$ Lukasiewicze; otherwise, $\tau: a_{i} \times a_{j} \rightarrow$ product:

$H_{6}>H_{1}>H_{3}>H_{5}>H_{4}>H_{2}$;

If $i, j \in\{1,2\}, \tau: a_{i} \times a_{j} \rightarrow$ Łukasiewicze; otherwise, $\tau: a_{i} \times a_{j} \rightarrow$ product):

$H_{6}>H_{3}>H_{1}>H_{4}>H_{2}>H_{5}$.

On the one hand, DMs need to consult relevant experts before making a decision. DMs may also represent explicit preferences among the attributes by associating different family adjoint triples. However, this preference is hard to quantify. In other words, how to choose different adjoint triples is difficult for DMs. At the same time, the calculation process is relatively complex. On the other hand, the IFNs of $H_{2}$ is lager than $H_{5}$ according to attributes $a_{1}, a_{2}$ and $a_{4}$. The IFN of $a_{3}$ for $H_{2}$ is nearly as the same as $H_{5}$. Intuitively, $H_{2}$ should be in front of $H_{5}$ all the ranked results. However, we get $H_{5}>H_{2}$ in the results of the first adjoint triple and the second adjoint triple above. As a matter of fact, our approach gets the order $H_{2}>H_{5}$, as desired.

\section{Conclusion and further work}

On the basis of analyzing the existing sorting methods of IFNs, we define the ideal measure for sorting IFNs by considering the fuzzy semantics expressed by the hesitation degree of IFNs and the Euclidean distance between IFNs with the ideal positive IFN and the ideal negative IFN. Some desirable properties have also been strictly checked, such as weak admissibility, membership degree robustness, nonmembership degree robustness and determinism. After that, a numerical example is applied to demonstrate the reasonability of the proposed measure. Moveover, we introduce the ranking method to acquire the optimal alternative in MADA problems. Comparison analysis are used to show the effectiveness and simplicity of the proposed method. In the following research, we will focus on how to apply ideal measurement to practical problems in a wide range.

Acknowledgements This paper is supported by the National Natural Science Foundation of China (No. 62076088) and by the Natural Science Foundation of Hebei Province (No. A2020208004).

\section{Authors' contribution:}

Mi J.S. directs and provides the conception of the work. Liang M.S. builds the measure, builds the applies, and drafts the article. 
Zhang S.P. and Jin C.X revise the article.

\section{Compliance with Ethical Standards:}

We declare that we have no conflict of interest. This article does not contain any studies with human participants or animals performed by any of the authors. Informed consent was obtained from all individual participants included in the study.

\section{References}

1. Arrow K J. Rational choice functions and orderings. Economica, 1959,102(26):121-127

2. Atanassov K. Intuitionistic fuzzy sets. Fuzzy Sets and Systems, 1986 20(1):87-96

3. Atanassov K. More on intuitionistic fuzzy sets. Fuzzy Sets and Systems, 1989, 33(1):37-45

4. Bustince H, Fernandez J, Kolesarova A, et al. Generation of linear orders for intervals by means of aggregation functions. Fuzzy Sets and Systems, 2013, 220(1):69-77

5. Chaira T. A novel intuitionistic fuzzy $\mathrm{C}$ means clustering algorithm and its application to medical images. Applied Soft Computing, 2011, 11(2):1711-1717

6. Chen S M, Tan J M. Handling multicriteria fuzzy decisionmaking problems based on vague set theory. Fuzzy Sets \& Systems, 1994, 67(2):163-172

7. Curley S P, Yates J F. The center and range of the probability interval as factors affecting ambiguity preferences. Organizational Behavior \& Human Decision Processes, 1985, 36(2):273-287

8. Das S, Guha D, Dutta B. Medical diagnosis with the aid of using fuzzy logic and intuitionistic fuzzy logic. Applied Intelligence, 2016, 45(3):850-867

9. Fei L G, Feng Y Q, Liu L N, et al. On intuitionistic fuzzy decision-making using soft likelihood functions. International Journal of Intelligent Systems, 2019, 34(1):22252242

10. Fu Q, Song Y, Fan C L, et al. Evidential model for intuitionistic fuzzy multi-attribute group decision making. Soft Computing, 2020, 24:7615-7635

11. Gandhimathi T. An Application of Intuitionistic Fuzzy Soft Matrix in Medical Diagnosis. Journal of Computational and Theoretical Nanoscience, 2018, 15(3):781-784

12. Grdenfors P, Sahlin N E. Unreliable probabilities, risk taking, and decision making. Synthese, 1982, 53(3):361-386

13. Guo K H. Amount of information and attitudinal-based method for ranking Atanassov's intuitionistic fuzzy values. IEEE Transactions on Fuzzy Systems, 2014, 22(1):177-188

14. He X X, Li Y F, Qin K Y, et al. Distance measures on intuitionistic fuzzy sets based on intuitionistic fuzzy dissimilarity functions. Soft Computing, 2020, 24:523-541

15. Hong D H, Choi C H. Multicriteria fuzzy decision-making problems based on vague set theory. Fuzzy Sets \& Systems, 2000, 114(1):103-113

16. Huang B, Li H X, Feng G F, et al. Double-quantitative rough sets, optimal scale selection and reduction in multiscale dominance IF decision tables. International Journal of Approximate Reasoning, 2021,130:170-191

17. Hrbacek K, Jech T. Introduction to Set Theory, Third Edition, Revised and Expanded. American mathematical monthly, 1999(1):100-100
18. Jiao L, Yang H L, Li S G. Three-way decision based on decision-theoretic rough sets with single-valued neutrosophic information. International Journal of Machine Learning and Cybernetics, 2020, 11(15):657-665

19. Kuo R J, Lin T C, Zulvia F E, et al. A Hybrid Metaheuristic and Kernel Intuitionistic Fuzzy c-means Algorith$\mathrm{m}$ for Cluster Analysis. Applied Soft Computing, 2018, 67:299-308

20. Liang M S, Mi J S, Feng T, Xie B. Multi-adjoint based group decision-making under an intuitionistic fuzzy information system. International Journal of Computational Intelligence Systems, 2018, 12(1):172-182

21. Liang M S, Mi J S, Zhao T N. Generalized dominancebased multi-granularity intuitionistic fuzzy rough set and acquisition of decision rules. CAAI Transactions on Intelligent Systems, 2017, 12(6):883-888

22. Liang M S, Mi J S, Feng T. Generalized dominancebased multigranulation intuitionistic fuzzy rough set and attribute reduction. Computer Science, 2018, 45(10):54-58

23. Liang M S, Mi J S, Feng T . Optimal granulation selection for similarity measure-based multigranulation intuitionistic fuzzy decision-theoretic rough sets. Journal of Intelligent \& Fuzzy Systems, 2019, 36(3):2495-2509

24. Qian J, Xin J, Shin-Jye L, et al. A new similarity/distance measure between intuitionistic fuzzy sets based on the transformed isosceles triangles and its applications to pattern recognition. Expert Systems with Applications, 2019, 116:439-453

25. Rajesh J, Satish K. Exponential Jensen intuitionistic fuzzy divergence measure with applications in medical investigation and pattern recognition. Soft Computing, 2019, 23(18):8995-9008

26. Szmidt E, Kacprzyk R. Amount of Information and Its Reliability in the Ranking of Atanassov's Intuitionistic Fuzzy Alternatives. Recent Advances in Decision Making. Springer Berlin Heidelberg, 2009

27. Xing Z J, Xiong W, Liu H. A Euclidean Approach for Ranking Atanassov Intuitionistic Fuzzy Values. IEEE Transactions on Fuzzy Systems, 2018, 26:353-365

28. Xu Z S. Multiple-attribute group decision making with different formats of preference information on attributes. IEEE Transactions on Systems Man \& Cybernetics Part B, 2007, 37(6):1500-1511

29. Xu Z S. Intuitionistic preference relations and their application in group decision making. Information Sciences, 2007, 177(11):2363-2379

30. Xu Z S, Yager R R. Some geometric aggregation operators based on intuitionistic fuzzy sets. International Journal of General Systems, 2006, 35(4):417-433

31. Zadeh L. Fuzzy sets. Information and Control, 1965, 8(3):338-353

32. Yates J F, Zukowski L G. Characterization of Ambiguity in Decision Making. Behavioral Science, 1976, 21(1):19-25

33. Zhang H D, Zhan J M, He Y P. Multi-granulation hesitant fuzzy rough sets and corresponding applications. Soft Computing, 2019, 23(1):13085-13103

34. Zhan J M, Malik H M, Akram M. Novel decision-making algorithms based on intuitionistic fuzzy rough environment. International Journal of Machine Learning \& Cybernetics, 2019, 10(6):1459-1485

35. Zhang X M, Xu Z S. A new method for ranking intuitionistic fuzzy values and its application in multi-attribute decision making. Fuzzy Optimization and Decision Making, 2012, 11(2):135-146

36. Zhao H, Xu Z S, Liu S S, et al. Intuitionistic fuzzy MST clustering algorithms. Computers \& Industrial Engineering, 2012, 62(4):1130-1140 
37. Zhao H L, Ma W M, Sun B Z. A novel decision making approach based on intuitionistic fuzzy soft sets. International Journal of Machine Learning and Cybernetics, 2017, 8(4):1107-1117

38. Wei Y, Jhang S T, Fu Z W, et al. A novel method to derive the intuitionistic fuzzy priority vectors from intuitionistic fuzzy preference relations. Soft Computing, 2021, 25:145-159 